\title{
Snacking patterns, diet quality, and cardiovascular risk factors in adults
}

Theresa A Nicklas ${ }^{1 * \dagger}$, Carol E O'Neil ${ }^{2+}$ and Victor L Fulgoni $\|^{3+}$

\begin{abstract}
Background: The relationship of snacking patterns on nutrient intake and cardiovascular risk factors (CVRF) in adults is unknown. The aim of this study was to examine the associations of snacking patterns with nutrient intake, diet quality, and a selection of CVRF in adults participating in the 2001-2008 National Health and Nutrition Examination Survey.

Methods: 24-hour dietary recalls were used to determine intake and cluster analysis was used to identify the snacking patterns. Height and weight were obtained and the health indices that were evaluated included diastolic and systolic blood pressure, high density lipoprotein-cholesterol, low density lipoprotein cholesterol, triacylglycerides, blood glucose, and insulin.
\end{abstract}

Results: The sample was participants ( $n=18,988)$ 19+ years ( $50 \%$ males; $11 \%$ African-Americans; $72 \%$ white, $12 \%$ Hispanic-Americans, and 5\% other). Cluster analyses generated 12 distinct snacking patterns, explaining $61 \%$ of the variance in snacking. Comparisons of snacking patterns were made to the no snack pattern. It was found that miscellaneous snacks constituted the most common snacking pattern (17\%) followed by cakes/cookies/pastries (12\%) and sweets (9\%). Most snacking patterns were associated with higher energy intakes. Snacking patterns cakes/cookies/pastries, vegetables/legumes, crackers/salty snacks, other grains and whole fruit were associated with lower intakes of saturated fatty acids. Added sugars intakes were higher in the cakes/cookies/pastries, sweets, milk desserts, and soft drinks patterns. Five snack patterns (cakes/cookies/pastries, sweets, vegetable/legumes, milk desserts, soft drinks) were associated with lower sodium intakes. Several snack patterns were associated with higher intakes of potassium, calcium, fiber, vitamin A, and magnesium. Five snacking patterns (miscellaneous snacks; vegetables/legumes; crackers/salty snacks; other grains; and whole fruit) were associated with better diet quality scores. Alcohol was associated with a lower body mass index and milk desserts were associated with a lower waist circumference. No snack patterns were associated with other CVRF studied.

Conclusions: Overall, several snacking patterns were associated with better diet quality than those consuming no snacks. Yet, the majority of the snacking patterns were not associated with CVRF. Education is needed to improve snacking patterns in terms of nutrients to limit in the diet along with more nutrient-dense foods to be included in snacks.

Keywords: Adults, Snacking patterns, Overweight, Abdominal obesity, Cardiovascular risk factors, Diet quality

\footnotetext{
* Correspondence: tnicklas@bcm.edu

${ }^{\dagger}$ Equal contributors

${ }^{1}$ Department of Pediatrics, Baylor College of Medicine, USDA/ARS Children's

Nutrition Research Center, 1100 Bates Ave, Houston, TX 77030, USA

Full list of author information is available at the end of the article
} 


\section{Background}

In 2009-2010, 36\% of adults in the US were obese [1]. Obesity increases the risk of a number of health conditions including hypertension, dyslipidemia, type 2 diabetes, and metabolic syndrome $[2,3]$. Obesity also affects quality of life, increases medical cost, and increases job absenteeism in adults [4-8]. The direct and indirect cost associated with obesity in adults is estimated at $\$ 209$ billion or $21 \%$ of US healthcare expenditures [9].

Snacking has become ubiquitous in the American society. The percentage of adults consuming snacks increased from $71 \%$ in $1977-1978$ to $97 \%$ in $2003-2006$ [10]. The number of snacking occasions increased 0.97 events over this same time period and the contribution of snacks to total energy intake increased from $18 \%$ to $24 \%$ [10]. Snacking has been shown to be associated with increased energy intake [11]. The increased energy intake associated with snacking may reflect the energy density [12] and portion sizes of many foods and beverages consumed as snacks [13-15]. Snacking also contributed significantly to nutrient intake $[11,16,17]$, better diet quality [18], and increased likelihood of meeting selected national recommendations $[11,16]$.

Studies have suggested that several characteristics of dietary behavior such as eating frequency or snacking [19-21] may influence body weight. The Booth Hypothesis [22] stated that "grazing" or multiple eating episodes between meals, rather than the traditional pattern of three meals per day, was a major factor that contributed to obesity. Contrary to this hypothesis, few adult studies have shown that snacking was positively [23] or negatively associated with body fatness $[19,24]$ or reduced risk of overweight and abdominal obesity $[25,26]$. Others have shown that snacking was not associated with weight [11,24,26-28] and was not an independent predictor of weight gain [29]. Results may be equivocal because snack definitions have not been clearly established, thus were not consistent across studies [12,30-32]. The snacking studies were also based on the assumption that snacking patterns were not unique in their contribution to nutrient intake. There are several possible explanations for the lack of association between snacking patterns and weight, despite the increased energy intake associated with the snacking patterns. Snacking has been shown to be associated with improved diet quality $[11,14,33]$ and increased intakes of fruit, whole grains, and fiber [11,14], which could promote satiety and reduce risks for obesity. Snacking has also been associated with increased vigorous physical activity [16,34]; thus, the increased energy intake associated with snacking may have been compensated for by increased energy expenditure during physical activity. A lack of association between snacking and weight could also be explained if overweight individuals who try to lose weight avoid eating snacks. More studies are needed to better understand the mechanisms by which snacking may impact the balance of energy intake and energy expenditure.

Few studies have attempted to examine the association of snacking with specific cardiometabolic risk factors [35-39]. Majority of these studies were limited to foreign populations of male and/or female adults or adolescents and snacks were not uniformly defined across these studies and were typically included as a component of food patterns or dietary recommendations. Snack foods were determined using a food frequency questionnaire with a limited number of snack foods assessed. None of these studies looked at snacking patterns, which can vary considerably, with cardiovascular risk factors (CVRF) in a nationally representative population of US adults. To our knowledge this is the first study to examine the various snacking patterns among adults and their impact on nutrient intake, diet quality, and a selection of CVRF (including overweight/obesity).

\section{Methods}

\section{Study overview, population, and analytic sample}

Data from adults $19+$ years of age $(y)(n=18,988)$ participating in the National Health and Nutrition Examination Survey (NHANES) 2001-2008 were combined for these analyses to increase the sample size [40]. This was a secondary data analysis with a lack of personal identifiers; therefore, this study was exempted by the Baylor College of Medicine Institutional Review Board.

\section{Dietary and physical activity assessment}

Dietary intake data were obtained from in-person 24-hour dietary recall interviews (Day 1) using an Automated Multiple-Pass Method [41] in the Mobile Examination Center (MEC). The Multiple-Pass Method [42-44] consisted of five steps: (a) the quick list, which was an uninterrupted listing by the subject of foods and beverages consumed; (b) the forgotten foods list, which queried the subject on categories of foods that have been documented as frequently forgotten; (c) a time and occasion at which foods were consumed; (d) the detail cycle, which elicited descriptions of foods and amounts eaten aided by the interactive use of a Food Model Booklet and measuring guides; and finally, (e) the final probe review. For data collection years 2001-2002, only a single 24-hour dietary recall was collected. Although two 24-hour dietary recalls were collected in 2003-2008, only data from the first recall was used to assess snacking patterns. There was a concern that differences in methodology might confound the results. Adults whose 24-hour recall data were judged to be incomplete or unreliable by staff of the National Center for Health Statistics (Hyattsville, MD) were excluded from these analyses. Females who were pregnant or lactating were also excluded. Snacks were self-defined by subjects as eating occasions with foods or beverages not consumed 
with meals. The timing of eating meals was defined in the 24-hour recall. Detailed descriptions of the dietary recalls and data collection are available in the NHANES Dietary Interviewer's Training Manual [45].

Energy and nutrient intakes were calculated using the USDA's Food and Nutrient Database for Dietary Studies (versions 1.0 - 4.1) [46], for NHANES 2001-2002, 20032004, 2005-2006, and 2007-2008. The nutrients studied reflect the nutrients to limit in the diet (i.e. total energy, saturated fatty acids, added sugars and sodium), nutrients of public health concern (i.e. potassium, calcium, vitamin $\mathrm{D}$, and fiber), and nutrients under-consumed (i.e. vitamin A, vitamin $\mathrm{C}$, vitamin $\mathrm{K}$, folate, and magnesium), as defined by the 2010 Dietary Guidelines for Americans [47]. The MyPyramid Equivalents Database (MPED), versions 1.0 [48] and 2.0 [49], was used to examine consumption in terms of MyPyramid [49] food group equivalents. The MPED translates dietary recall data into equivalent servings of the seven MyPyramid major food groups and corresponding subgroups. The number of MyPyramid food group equivalent servings was based on the 24-hr food dietary recall data from NHANES 2001-2008. Foods were hand matched to the same/similar foods for NHANES 2003-2008 since these data were released without an update to the MPED.

Diet quality was calculated using the Healthy Eating Index-2005 (HEI-2005) [50,51]. Food group standards and the development and evaluation of the HEI-2005 have been previously described [52,53]. The SAS code used to calculate HEI-2005 scores was downloaded from the Center for Nutrition Policy and Promotion website [54]. Briefly, HEI-2005 was designed to evaluate all of the major MyPyramid food groups and major subgroups. The 12 HEI-2005 components were summed for a total possible score of 100 points. Each participant's component score was calculated by dividing the total component intake by the total energy intake and multiplying by 1000. Scores were energy-adjusted on a density basis (per $4187 \mathrm{~kJ}$ ), which allowed for characterization of diet quality while controlling for diet quantity. Physical activity was determined using a questionnaire [55] that assessed sedentary, moderate and vigorous physical activity in a typical week.

\section{Physiologic measures}

Height and weight were obtained according to NHANES Anthropometry Procedures Manual [56]. The manual provides information about equipment, calibration, methods, quality control, and survey procedures. Anthropometry data was measured data by study researchers in NHANES. Body mass index was calculated as body weight (in kilograms) divided by height (in meters) squared [57]. Waist circumference (WC) was obtained using NHANES protocols [56].

\section{Cardiovascular risk factors}

Health indices that were evaluated included diastolic (DBP) and systolic blood pressure (SBP), high density lipoprotein-cholesterol (HDL-C), low density lipoprotein cholesterol (LDL-C), triacylglycerides (TAG), blood glucose, and insulin. Measurements of CVRF were obtained in the MEC according to the NHANES protocols $[56,58]$. Three or four readings for SBP and DBP were recorded in the NHANES; an average from each set of readings was used in this study. Venous blood was drawn in the MEC and total HDL-C were determined on non-fasted individuals $(\mathrm{n}=18,988)$ and LDL-C, TAG, and blood glucose were determined on only fasted subjects $(n=8,099)$; thus, not all individuals had laboratory values for all tests. Plasma glucose was measured spectrophotometrically using a series of enzymatic reactions (Roche Diagnostics, IN) [59]. Serum LDL-C was calculated according to the Friedewald equation and was reported only for fasting participants [59]. Serum HDL-C was measured using enzymatic reactions in conjunction with the heparinmanganese precipitation method or a direct immunoassay technique (Roche Diagnostics, IN) [59].

\section{Clinical definitions}

Overweight/obesity was defined as a BMI $\geq 25$ [60]; elevated waist circumference, WC $\geq 102 \mathrm{~cm}$ (males) or $\geq 88 \mathrm{~cm}$ (females); elevated blood pressure, $\mathrm{SBP} \geq 130 \mathrm{mmHg}$ or DBP $\geq 85 \mathrm{mmHg}$ or antihypertensive medication use; reduced serum HDL-C, $<2.22 \mathrm{mmol} / \mathrm{l}$ (males) or $<2.77 \mathrm{mmol} / \mathrm{l}$ (females) or medication use for reduced HDL-C; elevated serum $\mathrm{LDL}-\mathrm{C}, \geq 3.37 \mathrm{mmol} / \mathrm{l}$; elevated serum TAG, $\geq 8.33 \mathrm{mmol} / \mathrm{l}$ or medication use for elevated TAG; elevated fasting plasma or serum glucose, $\geq 5.55 \mathrm{mmol} / \mathrm{l}$ or medication use for elevated glucose. Data on medication use were obtained from the NHANES household interview on prescription medications or from questionnaires pertaining to blood pressure and diabetes mellitus. Abnormal values for other cardiovascular risk factors (CVRF) were determined using established criteria [61-65].

\section{Cluster analysis}

Snacking intake patterns were identified using SAS 9.2 (SAS Institute, Cary, NC, 2009) PROC CLUSTER using a single 24-hour dietary recall in NHANES 2001-2008. NHANES population weights were applied. Cluster analyses allow the user to focus on a particular defined aspect (e.g. snacking calories) and then forces maximal differences in clusters for assessment. Cluster analysis also allows for group comparisons rather than factor analysis which are generally associations. For these analyses, the USDA food groups were collapsed into 21 snacking food groupings. All food codes fit in one and only one of the snacking food groupings. The patterns identified by the cluster analysis were then identified by percent calories 
within each snacking food grouping (only foods that contributed $5 \%$ or more of calories were included) at the centroid of each cluster. Using this method resulted in 12 readily identifiable snacking patterns, such as crackers/salty snacks, sweets, fruit; no snacks was one of the 12 patterns identified. With snacking patterns identified, each participant was placed into one snacking pattern. A subject was placed in one of 12 snacking patterns based on the percent of calories from snacks for the day falling in each of the distinct food categories. The cluster definitions and the associations of subjects with a cluster are directly from the output from the cluster procedure and each subject was then placed in the cluster that matched most closely to the pattern of calories across the food categories.

\section{Statistical analyses}

For the initial analyses, SUDAAN v10.0 (Research Triangle Institute; Raleigh, NC) was used to adjust analyses for sampling weights and the sampling units and strata information as provided by NHANES. Dietary day 1 weights were used for all analyses. Least-square means \pm SE were calculated using PROC REGRESS of SUDAAN for dietary intake, diet quality (HEI-2005), and physiological measures were determined for participants consuming each snacking pattern. Covariates included age, gender, and race/ ethnicity. The poverty income ratio (PIR) grouped into three categories $(<1.25,1.25-3.49$, and $>3.49)$, physical activity level (sedentary, moderate and vigorous), alcohol intake $(\mathrm{g} / \mathrm{d})$, and energy intake for nutrient related variables (not for energy intake itself, HEI-2005, or physiological measures) also served as covariates. The PIR values reflected the federally set poverty lines, so a PIR of $<1.25$ equated to below $125 \%$ of poverty. Higher values mean the individuals had higher incomes. The HEI-2005 was not controlled for energy intake, since it is already controlled for energy [66]. Statistical differences for variables of interest were determined via $t$-test comparing to the no snacking group.

Odds ratios and Bonferroni corrected $95^{\text {th }}$ percentile upper and lower confidence intervals for being overweight, being obese, and having other cardiovascular risk factors were evaluated with no snacks as the reference group using the PROC LOGISTIC procedure of SUDAAN software controlling for the above mentioned covariates. A probability of $\mathrm{p} \leq 0.05$ was considered significant; however, a Bonferroni correction was applied for multiple comparisons $(0.05 / 12)$, so the effective $\mathrm{p}$ value was $\mathrm{p} \leq 0.0042$.

\section{Results}

\section{Demographics of the snacking patterns}

The demographic characteristics of the sample for each of the 12 snacking patterns are presented in Table 1 .
The total sample $(\mathrm{n}=18,988)$ was aged 19 y and older (50\% males; $11 \%$ African-Americans; $72 \%$ whites; $12 \%$ Hispanic-American and $5 \%$ other) with a mean poverty ratio of $3.02 ; 37 \%$ of the population reported doing moderate-vigorous physical activity. Thirteen percent reported consuming no snacks.

\section{Description of snacking patterns}

The 12 snacking patterns ( $\mathrm{n}$ and\% of population) were: miscellaneous snacks $(n=3,230,17 \%)$, which included fruit juice, fruit drinks, meat/poultry/fish, cheese, low-fat milk, cakes/cookies/pastries, and crackers/salty snacks; no snacks $(\mathrm{n}=2,853,13 \%)$; cakes/cookies/pastries $(\mathrm{n}=2,180,12 \%)$; sweets $(\mathrm{n}=1,495,9 \%)$; vegetables/legumes $(\mathrm{n}=1,524$, $8 \%)$; alcohol $(\mathrm{n}=1,572,8 \%)$; milk desserts $(\mathrm{n}=1,355,8 \%)$; crackers/salty snacks $(\mathrm{n}=1,293,7 \%)$; soft drinks $(\mathrm{n}=1,088$, $6 \%)$; other grains $(n=1,190,6 \%)$; whole fruit $(n=913,4 \%)$; and, coffee/tea $(n=295,2 \%)$. A description of the foods in the snacking pattern clusters is provided in Figure 1.

\section{Energy and nutrients to limit}

Table 2 shows mean intakes of energy and nutrients to limit, including saturated fatty acid (SFA), solid fat, added sugars, and sodium by snacking patterns for the entire day. Most of the snacking patterns were associated with a higher total energy intake, with the exception of the alcohol, whole fruit, and coffee/tea snacking patterns when compared to no snacks. Total energy intake of the snacking patterns ranged from 7,939 kJ (no snacks) to 10,153 kJ (other grains).

Total SFA intake of the snacking patterns ranged from 9.94 $\mathrm{g}$ (whole fruit) to $12.45 \mathrm{~g}$ (milk desserts). Total intake of SFA was significantly higher in the milk desserts snacking pattern and lower in the crackers/salty snacks, soft drinks, other grains and whole fruit snacking patterns, compared to no snacks. Total intake of solid fat was similar to SFA intake. Total intake of solid fat of the snacking patterns ranged from $39.5 \mathrm{~g}$ (vegetables/legumes) to $50.6 \mathrm{~g}$ (milk desserts).

Total intake of added sugars ranged from $15.1 \mathrm{~g}$ (whole fruit) to $28.7 \mathrm{~g}$ (soft drinks). Total intake of added sugars was significantly higher in the majority of the snacking patterns, except the snacking patterns, miscellaneous snacks, alcohol, and coffee/tea, compared to no snacks. Total intake of sodium ranged from 3,245 mg (milk desserts and soft drinks) to $3,753 \mathrm{mg}$ (crackers/salty snacks). Total intake of sodium was significantly lower in the cakes/cookies/ pastries, sweets, vegetables/legumes, milk desserts, and soft drinks snacking patterns, compared to no snacks.

\section{Nutrients of public health concern and nutrients under- consumed}

Table 3 shows the mean intake of nutrients of public health concern and nutrients that are of potential 
Table 1 Demographics of the sample by snacking patterns in adults $\geq 19$ years of age

\begin{tabular}{|c|c|c|c|c|c|c|c|c|c|c|c|c|c|}
\hline \multirow[b]{2}{*}{ Demographics \% } & \multirow[b]{2}{*}{$\begin{array}{c}\text { Total } \\
\text { sample } \\
(n=18,988)\end{array}$} & \multicolumn{12}{|c|}{ Snacking patterns } \\
\hline & & $\begin{array}{c}\text { Misc. } \\
\text { snacks } \\
(n=3,230)\end{array}$ & $\begin{array}{l}\text { No snacks** } \\
(n=2,853)\end{array}$ & $\begin{array}{c}\text { Cakes/ } \\
\text { Cookies/ } \\
\text { Pastries } \\
(\mathbf{n}=\mathbf{2}, 180)\end{array}$ & $\begin{array}{c}\text { Sweets } \\
(n=1,495)\end{array}$ & $\begin{array}{l}\text { Vegetables/ } \\
\text { Legumes } \\
(n=1,524)\end{array}$ & $\begin{array}{c}\text { Alcohol } \\
(n=1,572)\end{array}$ & $\begin{array}{c}\text { Milk } \\
\text { desserts } \\
(n=1,355)\end{array}$ & $\begin{array}{c}\text { Crackers/Salty } \\
\text { snacks } \\
(n=1,293)\end{array}$ & $\begin{array}{c}\text { Soft } \\
\text { drinks } \\
(n=1,088)\end{array}$ & $\begin{array}{c}\text { Other } \\
\text { grains } \\
(n=1,190)\end{array}$ & $\begin{array}{c}\text { Whole } \\
\text { fruit } \\
(n=913)\end{array}$ & $\begin{array}{c}\text { Coffee/Tea } \\
(n=295)\end{array}$ \\
\hline Total & 100 & 17 & 13 & 12 & 9 & 8 & 8 & 8 & 7 & 6 & 6 & 4 & 2 \\
\hline \multicolumn{14}{|l|}{ Gender } \\
\hline Male & 49 & 48 & 42 & 45 & 41 & 48 & 67 & 48 & 44 & 56 & 50 & 44 & 48 \\
\hline Female & 51 & 52 & 48 & 55 & 59 & 52 & 33 & 52 & 56 & 44 & 50 & 56 & 52 \\
\hline \multicolumn{14}{|l|}{ Ethnicity } \\
\hline White & 72 & 67 & 64 & 78 & 75 & 76 & 74 & 82 & 77 & 74 & 64 & 63 & 75 \\
\hline African-American & 11 & 14 & 13 & 11 & 10 & 10 & 13 & 8 & 11 & 10 & 11 & 10 & 6 \\
\hline Hispanic-American & 12 & 13 & 17 & 8 & 10 & 9 & 9 & 7 & 9 & 11 & 19 & 20 & 10 \\
\hline Other & 5 & 6 & 6 & 4 & 5 & 5 & 4 & 4 & 3 & 5 & 5 & 7 & 9 \\
\hline \multicolumn{14}{|l|}{ Physical Act. } \\
\hline Sedentary & 31 & 31 & 36 & 31 & 30 & 28 & 26 & 31 & 28 & 34 & 29 & 31 & 29 \\
\hline Light & 33 & 31 & 33 & 35 & 31 & 36 & 31 & 33 & 33 & 29 & 33 & 34 & 32 \\
\hline Mod-Vig & 37 & 37 & 32 & 34 & 39 & 36 & 44 & 36 & 39 & 37 & 38 & 35 & 39 \\
\hline$P I R^{*}$ & 3 & 3 & 3 & 3 & 3 & 3 & 3 & 3 & 3 & 3 & 3 & 3 & 3 \\
\hline
\end{tabular}

${ }^{*} \mathrm{PIR}=$ Poverty Income Ratio.

**Reference group in No Snacks. 


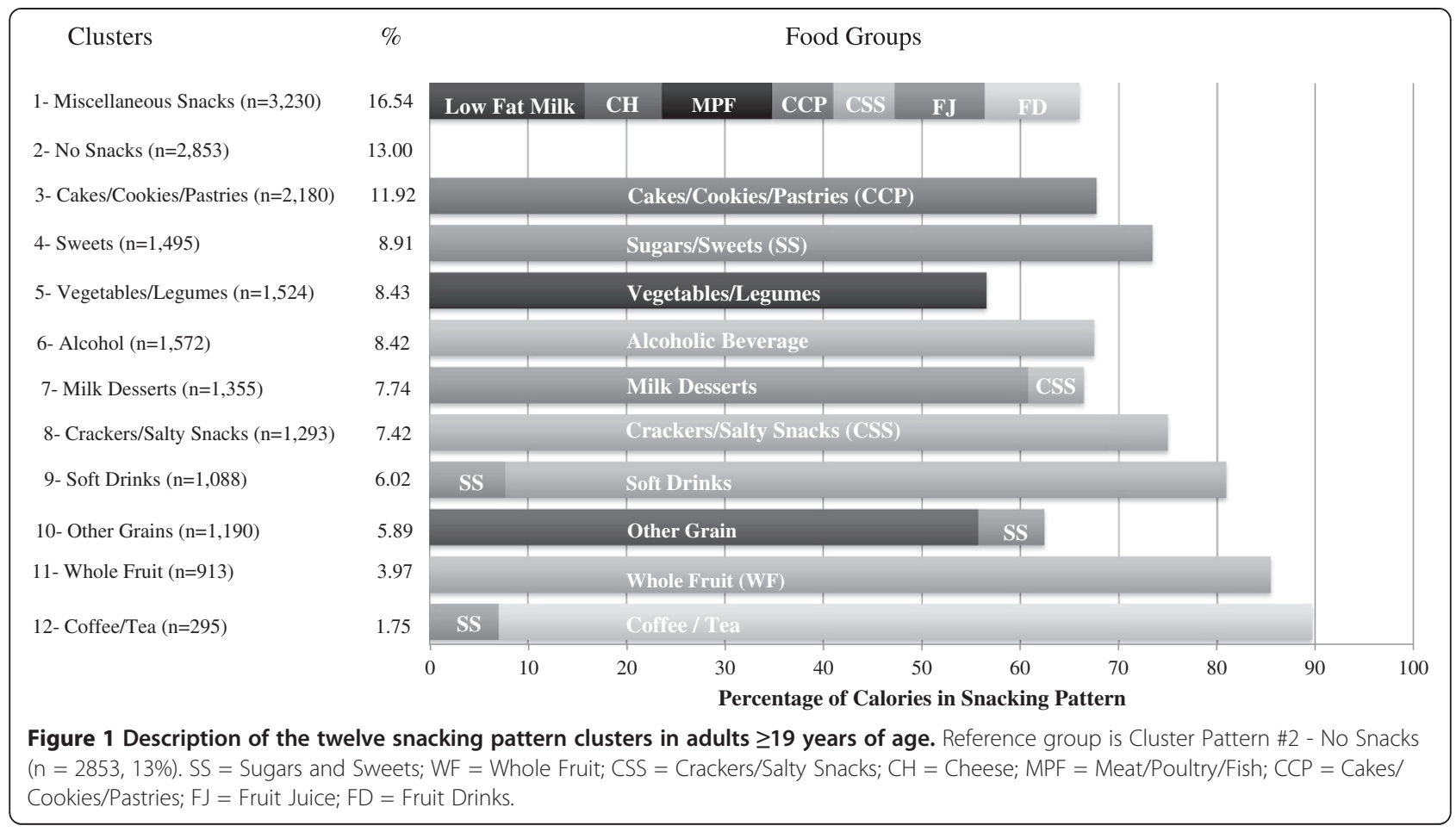

concern for under-consumption for sub-populations for the entire day. Total potassium intake ranged from $2,383 \mathrm{mg}$ (soft drinks) to $3,047 \mathrm{mg}$ (whole fruit). Total potassium intake was significantly higher in the miscellaneous snacks, vegetables/legumes, alcohol, milk desserts, soft drinks, whole fruit, and coffee/tea snacking patterns, compared to no snacks. Total calcium intake ranged from $788 \mathrm{mg}$ (soft drinks) to $1,043 \mathrm{mg}$ (miscellaneous snacks). Compared to no snacks, total calcium intake was higher for the miscellaneous snacks and lower for the soft drinks snacking patterns. Total intake of vitamin D ranged from $3.86 \mathrm{mcg}$ (crackers/salty snacks) to $5.50 \mathrm{mcg}$ (miscellaneous snacks). When vitamin D intake among the patterns was compared to no snacks, vitamin $D$ intake was higher for the miscellaneous snacks only. Total dietary fiber intake ranged from 13.6 gm (soft drinks) to $19.5 \mathrm{gm}$ (whole fruit). Total mean intake of dietary fiber was higher among the vegetables/ legumes, crackers/salty snacks, other grains and whole fruit snacking patterns compared to no snacks. For those in the soft drink pattern, total dietary fiber was lower than no snacks. Total intake of vitamin A was significantly higher in the miscellaneous snacks, alcohol, and milk desserts snacking patterns, compared to no snacks. Intake of vitamin $\mathrm{C}$ was higher in the miscellaneous snacks and whole fruit and lower in the soft drinks snacking patterns, compared to no snacks. Total intake of folate was higher in the other grains and lower in the soft drink snacking pattern, compared to no snacks. Total intake of magnesium was higher in the vegetables/legumes, crackers/salty snacks, whole fruit, and coffee/tea but lower in the soft drink snacking pattern, compared to no snacks.

\section{Overall diet quality}

Figure 2 shows the Healthy Eating Index-2005 (HEI-2005) by snacking patterns. On average the HEI-2005 scores were higher for five of the snacking patterns, miscellaneous snacks, vegetables/legumes, crackers/salty snacks, other grains, and whole fruit, compared to no snacks. The HEI-2005 score for the soft drink snacking pattern was lower than no snacks. Half of the snacking patterns had a total HEI-2005 score of less than 50; which included no snacks, cakes/cookies/pastries, sweets, alcohol, milk desserts, and soft drinks.

\section{Snacking patterns, weight/adiposity status, and cardiovascular risk factors}

Table 4 shows the relationship between the snacking patterns and weight/adiposity. Body mass index and WC were significantly lower in the alcohol and milk desserts snacking patterns respectively, compared to no snacks. For the remaining 10 snacking patterns, there was no association found with weight/adiposity. The percent of overweight/obese adults who reported $>30 \%$ of energy from snacks was significantly lower compared to normal weight adults (Additional file 1: Figure S1). None of the snacking patterns showed an increased likelihood of having elevated cardiovascular risk factors when compared to no snacks (Table 5). 
Table 2 Consumption of energy and nutrients to limit by snacking patterns ${ }^{1}$ in adults $\geq 19$ years of age

\begin{tabular}{|c|c|c|c|c|c|c|c|c|c|c|c|c|}
\hline \multirow[b]{2}{*}{$\begin{array}{l}\text { Energy \& } \\
\text { nutrients } \\
\text { to limit }\end{array}$} & \multicolumn{12}{|c|}{ Snacking Patterns LSM (SE) } \\
\hline & $\begin{array}{c}\text { Misc. } \\
\text { snacks } \\
(n=3,230)\end{array}$ & $\begin{array}{l}\text { No snacks** } \\
(n=2,853)\end{array}$ & $\begin{array}{c}\text { Cakes/ } \\
\text { Cookies/ } \\
\text { Pastries } \\
(n=2,180)\end{array}$ & $\begin{array}{c}\text { Sweets } \\
(n=1,495)\end{array}$ & $\begin{array}{c}\text { Vegetables/ } \\
\text { Legumes } \\
(n=1,524)\end{array}$ & $\begin{array}{c}\text { Alcohol } \\
(n=1,572)\end{array}$ & $\begin{array}{c}\text { Milk } \\
\text { desserts } \\
(\mathrm{n}=1,355)\end{array}$ & $\begin{array}{c}\text { Crackers/ } \\
\text { Salty } \\
\text { snacks } \\
(n=1,293)\end{array}$ & $\begin{array}{c}\text { Soft } \\
\text { drinks } \\
(n=1,088)\end{array}$ & $\begin{array}{c}\text { Other } \\
\text { grains } \\
(n=1,190)\end{array}$ & $\begin{array}{c}\text { Whole } \\
\text { fruit } \\
(n=913)\end{array}$ & $\begin{array}{c}\text { Coffee/ } \\
\text { Tea } \\
(n=295)\end{array}$ \\
\hline $\begin{array}{l}\text { Total } \\
\text { energy }(\mathrm{kJ})^{1}\end{array}$ & $\begin{array}{c}9538.66 \\
(100.07)^{*}\end{array}$ & $\begin{array}{l}7936.84 \\
(107.69)\end{array}$ & $\begin{array}{c}9959.07 \\
(104.13)^{*}\end{array}$ & $\begin{array}{c}9301.59 \\
(135.45)^{*}\end{array}$ & $\begin{array}{c}9812.11 \\
(134.32)^{*}\end{array}$ & $\begin{array}{l}8206.73 \\
(145.58)\end{array}$ & $\begin{array}{c}9767.43 \\
(145.75)^{*}\end{array}$ & $\begin{array}{c}8968.55 \\
(130.09)^{*}\end{array}$ & $\begin{array}{l}8908.05 \\
(179.45)\end{array}$ & $\begin{array}{l}10152.81 \\
(205.96)^{*}\end{array}$ & $\begin{array}{l}8083.38 \\
(146.63)\end{array}$ & $\begin{array}{l}8455.73 \\
(287.48)\end{array}$ \\
\hline $\begin{array}{l}\text { Saturated } \\
\text { fatty } \\
\text { acid }(g)^{1}\end{array}$ & $27.59(0.27)$ & $28.03(0.27)$ & $26.61(0.30)^{*}$ & $28.55(0.32)$ & $26.37(0.32)^{*}$ & $27.60(0.39)$ & $30.70(0.39)^{*}$ & $26.02(0.29)^{*}$ & $25.74(0.42)^{*}$ & $25.80(0.45)^{*}$ & $25.98(0.21)^{*}$ & $28.37(0.99)$ \\
\hline Solid fat $(g)^{1}$ & $46.91(0.52)$ & $49.05(0.65)$ & $49.83(0.57)$ & $45.83(0.61)^{*}$ & $39.49(0.74)^{*}$ & $47.82(0.78)$ & $50.64(0.67)$ & $45.11(0.75)^{*}$ & $44.97(0.81)^{*}$ & $45.22(0.96)^{*}$ & $44.95(0.89)^{*}$ & $47.87(1.43)$ \\
\hline $\begin{array}{l}\text { Added } \\
\text { sugars (tsp.) }{ }^{1}\end{array}$ & $17.94(0.40)$ & $18.68(0.51)$ & $23.17(0.52)^{*}$ & $22.77(0.50)^{*}$ & $15.61(0.58)^{*}$ & $18.93(0.59)$ & $21.58(0.55)^{*}$ & $16.66(0.51)^{*}$ & $28.74(0.73)^{*}$ & $16.35(0.56)^{*}$ & $15.13(0.46)^{*}$ & $18.63(1.08)$ \\
\hline $\begin{array}{l}\text { Sodium } \\
(\mathrm{mg})^{1}\end{array}$ & $\begin{array}{c}3568.88 \\
(27.24)\end{array}$ & $\begin{array}{c}3626.67 \\
(36.25)\end{array}$ & $\begin{array}{l}3321.33 \\
(35.87)^{*}\end{array}$ & $\begin{array}{l}3359.26 \\
(41.83)^{*}\end{array}$ & $\begin{array}{l}3400.26 \\
(44.34)^{*}\end{array}$ & $\begin{array}{c}3537.85 \\
(46.27)\end{array}$ & $\begin{array}{l}3245.11 \\
(44.52)^{*}\end{array}$ & $\begin{array}{c}3752.89 \\
(49.17)\end{array}$ & $\begin{array}{l}3245.54 \\
(46.25)^{*}\end{array}$ & $3638.47(57.04)$ & $\begin{array}{c}3612.98 \\
(44.20)\end{array}$ & $\begin{array}{l}3666.26 \\
(129.71)\end{array}$ \\
\hline
\end{tabular}

${ }^{1} \mathrm{kj}=$ kilojoules $(1 \mathrm{kcal}=4.187 \mathrm{kj})$. Covariates: Age, gender, race/ethnicity, poverty income ratio grouped into three categories as $(<1.25,1.25-3.49$, and $>3.49)$, current smoking status (yes/no), physical activity level (sedentary, moderate and vigorous), alcohol intake $(\mathrm{g} / \mathrm{d})$, energy intake for nutrient related variables.

*Statistically different from No Snacks; with the Bonferroni correction effective $p \leq 0.0042$.

**Reference group is No Snacks. 
Table 3 Consumption of nutrients of public health concern and nutrients under-consumed by snacking patterns ${ }^{1}$ in adults $\geq 19$ years of age

\begin{tabular}{|c|c|c|c|c|c|c|c|c|c|c|c|c|}
\hline & \multicolumn{12}{|c|}{ Snacking patterns LSM (SE) } \\
\hline & $\begin{array}{l}\text { Misc. snacks } \\
(n=3,230)\end{array}$ & $\begin{array}{l}\text { No snacks** } \\
(n=2,853)\end{array}$ & $\begin{array}{c}\text { Cakes/ } \\
\text { Cookies/ } \\
\text { Pastries } \\
(n=2,180)\end{array}$ & $\begin{array}{c}\text { Sweets } \\
(n=1,495)\end{array}$ & $\begin{array}{l}\text { Vegetables/ } \\
\text { Legumes } \\
(n=1,524)\end{array}$ & $\begin{array}{c}\text { Alcohol } \\
(n=1,572)\end{array}$ & $\begin{array}{c}\text { Milk } \\
\text { desserts } \\
(\mathrm{n}=1,355)\end{array}$ & $\begin{array}{l}\text { Crackers/ } \\
\text { Salty } \\
\text { snacks } \\
(n=1,293)\end{array}$ & $\begin{array}{c}\text { Soft } \\
\text { drinks } \\
(n=1,088)\end{array}$ & $\begin{array}{c}\text { Other } \\
\text { grains } \\
(n=1,190)\end{array}$ & $\begin{array}{c}\text { Whole } \\
\text { fruit } \\
(n=913)\end{array}$ & $\begin{array}{c}\text { Coffee/ } \\
\text { Tea } \\
(n=295)\end{array}$ \\
\hline \multicolumn{13}{|c|}{ Nutrients of Public Health Concern } \\
\hline Potassium (mg) & $\begin{array}{l}2883.71 \\
(27.34)^{*}\end{array}$ & $\begin{array}{c}2612.52 \\
(27.22)\end{array}$ & $\begin{array}{c}2521.52 \\
(23.69)\end{array}$ & $\begin{array}{c}2633.29 \\
(29.51)\end{array}$ & $\begin{array}{l}3028.60 \\
(37.96)^{*}\end{array}$ & $\begin{array}{l}2773.98 \\
(34.49)^{*}\end{array}$ & $\begin{array}{l}2765.11 \\
(39.83)^{*}\end{array}$ & $\begin{array}{c}2568.00 \\
(34.26)\end{array}$ & $\begin{array}{l}2383.33 \\
(30.03)^{*}\end{array}$ & $\begin{array}{c}2660.57 \\
(47.52)\end{array}$ & $\begin{array}{l}3047.08 \\
(45.95)^{*}\end{array}$ & $\begin{array}{l}2876.64 \\
(55.98)^{*}\end{array}$ \\
\hline Calcium (mg) & $\begin{array}{l}1043.12 \\
(13.55)^{*}\end{array}$ & $\begin{array}{l}892.64 \\
(13.40)\end{array}$ & $\begin{array}{l}854.50 \\
(13.01)\end{array}$ & $\begin{array}{l}852.62 \\
(12.22)\end{array}$ & 902.69 (20.99) & $901.23(18.02)$ & $949.75(16.76)$ & $\begin{array}{l}842.08 \\
(17.94)\end{array}$ & $\begin{array}{l}788.49 \\
(21.91)^{*}\end{array}$ & $\begin{array}{l}939.26 \\
(22.54)\end{array}$ & $934.96(22.46)$ & $958.84(34.72)$ \\
\hline Vitamin D (mcg) & $5.50(0.15)^{*}$ & $4.12(0.14)$ & $4.15(0.15)$ & $3.92(0.13)$ & $4.69(0.20)$ & $4.41(0.20)$ & $4.20(0.15)$ & $3.86(0.19)$ & $3.89(0.22)$ & $4.84(0.33)$ & $4.92(0.32)$ & $5.18(0.47)$ \\
\hline Fiber (gm) & $15.56(0.19)$ & $14.98(0.24)$ & $15.02(0.24)$ & $15.58(0.30)$ & $18.80(0.37)^{*}$ & $15.28(0.31)$ & $15.40(0.32)$ & $16.77(0.33)^{*}$ & $13.55(0.32)$ & $16.49(0.38)^{*}$ & $19.48(0.41)^{*}$ & $16.22(0.87)$ \\
\hline \multicolumn{13}{|c|}{ Nutrients under-consumed } \\
\hline Vitamin A (mcg) & $\begin{array}{c}656.07 \\
(12.68)^{*}\end{array}$ & $\begin{array}{l}577.56 \\
(12.86)\end{array}$ & $\begin{array}{l}630.15 \\
(19.46)\end{array}$ & $\begin{array}{l}561.44 \\
(17.36)\end{array}$ & $654.57(35.65)$ & $\begin{array}{l}615.13 \\
(19.82)^{*}\end{array}$ & $\begin{array}{l}681.73 \\
(18.63)^{*}\end{array}$ & $\begin{array}{l}583.58 \\
(18.97)\end{array}$ & $529.05(35.54)$ & $\begin{array}{l}651.46 \\
(60.74)\end{array}$ & $646.81(23.89)$ & $650.25(32.91)$ \\
\hline Vitamin C (mcg) & $109.10(3.01)^{*}$ & $84.34(2.69)$ & $81.62(2.54)$ & $81.39(3.82)$ & $87.50(3.55)$ & $81.61(3.06)$ & $87.58(4.06)$ & $79.93(3.48)$ & $68.26(3.24)^{*}$ & $86.92(5.35)$ & $123.05(5.03)^{*}$ & $74.40(4.07)$ \\
\hline Vitamin K (mcg) & $95.34(3.22)$ & $94.33(4.10)$ & $92.44(4.90)$ & $91.61(4.23)$ & $117.49(7.21)$ & $101.43(7.55)$ & $84.15(4.97)$ & $90.63(5.35)$ & $80.85(4.88)$ & $96.54(4.47)$ & $120.76(9.29)$ & $111.13(11.48)$ \\
\hline Folate (mcg) & $552.67(8.58)$ & $536.50(9.96)$ & $\begin{array}{l}546.60 \\
(11.73)\end{array}$ & $\begin{array}{l}504.67 \\
(11.16)\end{array}$ & $555.53(12.32)$ & 547.01 (13.91) & $531.35(15.69)$ & $\begin{array}{l}553.03 \\
(11.52)\end{array}$ & $\begin{array}{l}473.24 \\
(10.45)^{*}\end{array}$ & $\begin{array}{l}617.87 \\
(19.54)\end{array}$ & $572.67(13.40)$ & $547.89(20.17$ \\
\hline Magnesium (mg) & $299.13(2.63)$ & $276.51(2.80)$ & $269.62(2.95)$ & $277.84(3.46)$ & $360.62(7.46)^{*}$ & $290.43(4.56)$ & $282.62(3.86)$ & $\begin{array}{l}292.21 \\
(3.62)^{*}\end{array}$ & $245.69(3.32)^{*}$ & $287.32(4.72)$ & $307.46(5.58)^{*}$ & $\begin{array}{l}327.46 \\
(11.89)^{*}\end{array}$ \\
\hline
\end{tabular}

${ }^{1}$ Covariates: Age, gender, race/ethnicity, poverty income ratio grouped into three categories as ( $<1.25,1.25-3.49$, and $\left.>3.49\right)$, current smoking status (yes/no) (adults only), physical activity level (sedentary, moderate and vigorous), alcohol intake ( $\mathrm{g} / \mathrm{d})$, energy intake for nutrient related variables.

*Statistically different from No Snacks with the Bonferroni correction effective $p \leq 0.0042$.

**Reference group is No Snacks. 


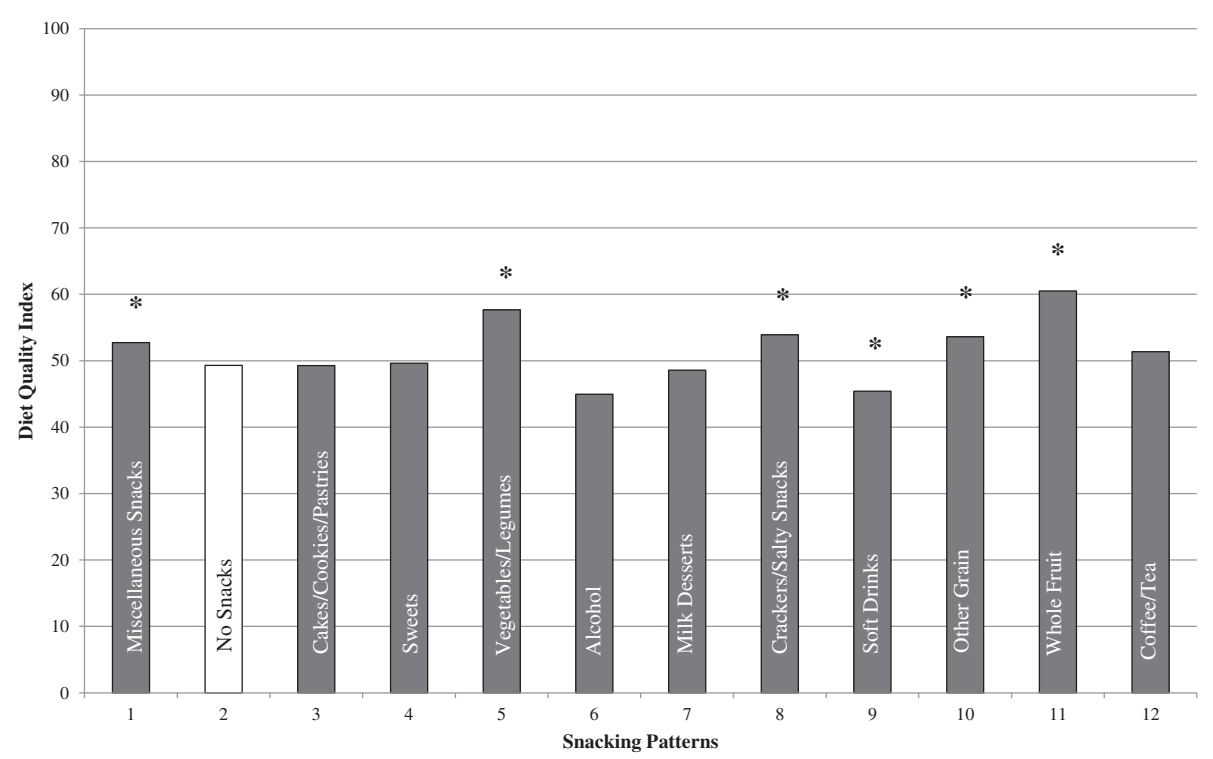

Figure 2 Snacking patterns and overall diet quality (HEI-2005) for adults $\geq 19$ years of age. Covariates: Age, gender, race/ethnicity, poverty income ratio grouped into three categories as $(<1.25,1.25-3.49$, and $>3.49)$, current smoking status yes/no) (adults only), physical activity level (sedentary, moderate and vigorous), alcohol intake ( $\mathrm{g} / \mathrm{d})$. Note that energy was not used as a covariate since the HEl score itself is controlled for energy. ${ }^{*}$ Significantly different from no snacking (pattern 2); Bonferroni correction $p \leq 0.0042$.

\section{Discussion}

Nutrition research has traditionally focused on single nutrients in relation to health. Recently however, scientists have acknowledged the complex synergistic interactions among foods in relation to health. This has led to a growing interest in looking at dietary patterns [67-73]

Table 4 Relationship between snacking patterns and weight by adults $\geq 19$ years of age

\begin{tabular}{llc}
\hline & \multicolumn{2}{c}{ Weight status ${ }^{\mathbf{1}}$} \\
\cline { 2 - 3 } Snacking pattern & BMI LSM (SE) & $\begin{array}{c}\text { Waist circumference, } \\
\text { cm LSM (SE) }\end{array}$ \\
\hline Miscellaneous snacks $(n=3230)$ & $28.39(0.19)$ & $97.10(0.47)$ \\
No snacks $(n=2853)$ & $28.54(0.18)$ & $97.66(0.48)$ \\
Cakes/Cookies/Pastries & $28.23(0.16)$ & $97.66(0.48)$ \\
( $=2180)$ & & $96.80(0.43)$ \\
Sweets $(n=1495)$ & $28.20(0.23)$ & $96.92(0.60)$ \\
Vegetables/Legumes $(n=1524)$ & $28.34(0.26)$ & $97.12(0.63)$ \\
Alcohol $(n=1572)$ & $27.74(0.20)^{\S}$ & $95.72(0.49)^{\S}$ \\
Milk desserts $(n=1355)$ & $28.30(0.24)$ & $97.91(0.49)$ \\
Crackers/Salty snacks $(n=1293)$ & $28.49(0.21)$ & $97.78(0.65)$ \\
Soft drinks $(n=1088)$ & $28.45(0.25)$ & $97.20(0.63)$ \\
Other grains $(n=1190)$ & $28.24(0.29)$ & $96.89(0.77)$ \\
Whole fruit $(n=913)$ & $28.38(0.38)$ & $98.24(1.26)$ \\
Coffee/Tea $(n=295)$ & $29.18(0.68)$ & \\
\hline
\end{tabular}

${ }^{1}$ Covariates included age, gender, race/ethnicity, poverty income ratio, current smoking status, alcohol intake, and physical activity.

${ }^{\S}$ Statistically different from No Snacks with the Bonferroni correction effective $\mathrm{p} \leq 0.0042$. which makes intuitive sense, given that foods are generally not eaten in isolation. Thus, eating patterns may have a greater impact on metabolic risk factors than any single food, food group, or nutrient. This study showed that, using cluster analysis, 12 specific snacking patterns, including no snacks, could be identified in a nationally representative sample of US adults. The snacking patterns varied widely by foods consumed, nutrient contribution, and overall diet quality.

In our study, total energy intake varied by snacking pattern. Most of the snacking patterns resulted in a higher total energy intake compared to no snacks. This is consistent with other studies showing that snacking was associated with increased energy intake [11]. Three snacking patterns, alcohol, whole fruit, and coffee/tea, resulted in lower total energy intake compared to no snacks. Studies have shown that alcohol [74] or coffee/tea intake [75] was associated with lower weight or less weight gain. Other studies have shown that fruit intake was inversely associated with weight in African-American females [76,77], suggesting a lower total energy intake or increased physical activity.

Total intake of SFA was lowest for the whole fruit snacking pattern and highest for the milk desserts snacking pattern. Total intake of added sugars was significantly higher in the majority of the snacking patterns, compared to no snacks. The majority of the snacking patterns exceeded the recommendation of less than $10 \%$ of total energy from SFA [47]; none of the snacking patterns showed a mean daily intake of added sugars that exceeded 
Table 5 Likelihood of having cardiovascular risk factors

\begin{tabular}{|c|c|c|c|c|c|c|c|c|c|c|c|}
\hline \multirow[b]{2}{*}{$\begin{array}{l}\mathrm{CVRF}^{+} \text {odds } \\
\text { ratio }(\mathrm{LCL}, \mathrm{UCL})^{* *}\end{array}$} & \multicolumn{11}{|c|}{ Snacking patterns* } \\
\hline & $\begin{array}{l}\text { Misc. snacks } \\
(\mathrm{n}=3,230)\end{array}$ & $\begin{array}{c}\text { Cakes/ } \\
\text { Cookies/ } \\
\text { Pastries } \\
(\mathbf{n}=\mathbf{2}, \mathbf{1 8 0})\end{array}$ & $\begin{array}{l}\text { Sweets } \\
(n=1,495)\end{array}$ & $\begin{array}{c}\text { Vegetables/ } \\
\text { Legumes } \\
(n=1,524)\end{array}$ & $\begin{array}{c}\text { Alcohol } \\
(n=1,572)\end{array}$ & $\begin{array}{c}\text { Milk } \\
\text { desserts } \\
(n=1,355)\end{array}$ & $\begin{array}{c}\text { Crackers/ } \\
\text { Salty } \\
\text { snacks } \\
(n=1,293)\end{array}$ & $\begin{array}{c}\text { Soft } \\
\text { drinks } \\
(n=1,088)\end{array}$ & $\begin{array}{c}\text { Other } \\
\text { grains } \\
(n=1,190)\end{array}$ & $\begin{array}{c}\text { Whole } \\
\text { fruit } \\
(n=913)\end{array}$ & $\begin{array}{c}\text { Coffee/Tea } \\
(n=295)\end{array}$ \\
\hline Elevated BP & $\begin{array}{c}1.04 \\
(0.82,1.31)\end{array}$ & $\begin{array}{c}0.89 \\
(0.68,1.16)\end{array}$ & $\begin{array}{c}1.02 \\
(0.76,1.37)\end{array}$ & $\begin{array}{c}1.09 \\
(0.77,1.53)\end{array}$ & $\begin{array}{c}0.85 \\
(0.59,1.23)\end{array}$ & $\begin{array}{c}1.04 \\
(0.78,1.39)\end{array}$ & $\begin{array}{c}1.13 \\
(0.79,1.61)\end{array}$ & $\begin{array}{c}1.09 \\
(0.75,1.60)\end{array}$ & $\begin{array}{c}0.98 \\
(0.67,1.43)\end{array}$ & $\begin{array}{c}0.94 \\
(0.64,1.37)\end{array}$ & $\begin{array}{c}0.97 \\
(0.58,1.61)\end{array}$ \\
\hline Elevated Glucose & $\begin{array}{c}1.08 \\
(0.75,1.55)\end{array}$ & $\begin{array}{c}1.06 \\
(0.74,1.52)\end{array}$ & $\begin{array}{c}0.87 \\
(0.57,1.34)\end{array}$ & $\begin{array}{c}1.34 \\
(0.96,1.88)\end{array}$ & $\begin{array}{c}0.86 \\
(0.56,1.34)\end{array}$ & $\begin{array}{c}1.16 \\
(0.78,1.71)\end{array}$ & $\begin{array}{c}1.01 \\
(0.63,1.60)\end{array}$ & $\begin{array}{c}0.89 \\
(0.55,1.45)\end{array}$ & $\begin{array}{c}1.30 \\
(0.81,2.09)\end{array}$ & $\begin{array}{c}1.30 \\
(0.73,2.32)\end{array}$ & $\begin{array}{c}0.58 \\
(0.31,1.11)\end{array}$ \\
\hline Elevated LDL-C & $\begin{array}{c}0.84 \\
(0.61,1.16)\end{array}$ & $\begin{array}{c}1.02 \\
(0.71,1.45)\end{array}$ & $\begin{array}{c}0.99 \\
(0.70,1.40)\end{array}$ & $\begin{array}{c}1.12 \\
(0.80,1.58)\end{array}$ & $\begin{array}{c}0.84 \\
(0.53,1.33)\end{array}$ & $\begin{array}{c}0.97 \\
(0.65,1.44)\end{array}$ & $\begin{array}{c}0.87 \\
(0.56,1.36)\end{array}$ & $\begin{array}{c}1.13 \\
(0.70,1.82)\end{array}$ & $\begin{array}{c}0.90 \\
(0.53,1.52)\end{array}$ & $\begin{array}{c}0.93 \\
(0.61,1.42)\end{array}$ & $\begin{array}{c}1.04 \\
(0.53,2.02)\end{array}$ \\
\hline Decreased HDL-C & $\begin{array}{c}0.96 \\
(0.77,1.19)\end{array}$ & $\begin{array}{c}0.94 \\
(0.74,1.20)\end{array}$ & $\begin{array}{c}1.23 \\
(0.95,1.59)\end{array}$ & $\begin{array}{c}0.89 \\
(0.68,1.17)\end{array}$ & $\begin{array}{c}0.88 \\
(0.60,1.29)\end{array}$ & $\begin{array}{c}0.97 \\
(0.73,1.30)\end{array}$ & $\begin{array}{c}1.08 \\
(0.85,1.37)\end{array}$ & $\begin{array}{c}1.40 \\
(0.98,1.99)\end{array}$ & $\begin{array}{c}0.94 \\
(0.68,1.31)\end{array}$ & $\begin{array}{c}1.08 \\
(0.81,1.44)\end{array}$ & $\begin{array}{c}1.15 \\
(0.63,2.11)\end{array}$ \\
\hline Elevated TAG & $\begin{array}{c}1.00 \\
(0.73,1.37)\end{array}$ & $\begin{array}{c}0.89 \\
(0.64,1.23)\end{array}$ & $\begin{array}{c}1.10 \\
(0.75,1.63)\end{array}$ & $\begin{array}{c}0.97 \\
(0.67,1.40)\end{array}$ & $\begin{array}{c}0.89 \\
(0.58,1.36)\end{array}$ & $\begin{array}{c}0.85 \\
(0.61,1.19)\end{array}$ & $\begin{array}{c}1.09 \\
(0.72,1.65)\end{array}$ & $\begin{array}{c}1.29 \\
(0.85,1.98)\end{array}$ & $\begin{array}{c}1.00 \\
(0.62,1.60)\end{array}$ & $\begin{array}{c}0.89 \\
(0.58,1.36)\end{array}$ & $\begin{array}{c}0.94 \\
(0.45,1.93)\end{array}$ \\
\hline Elevated WC & $\begin{array}{c}0.95 \\
(0.77,1.17)\end{array}$ & $\begin{array}{c}0.87 \\
(0.67,1.13)\end{array}$ & $\begin{array}{c}0.93 \\
(0.70,1.25)\end{array}$ & $\begin{array}{c}0.88 \\
(0.61,1.26)\end{array}$ & $\begin{array}{c}0.74 \\
(0.55,1.00)\end{array}$ & $\begin{array}{c}0.92 \\
(0.70,1.21)\end{array}$ & $\begin{array}{c}0.99 \\
(0.75,1.29)\end{array}$ & $\begin{array}{c}1.03 \\
(0.75,1.41)\end{array}$ & $\begin{array}{c}1.04 \\
(0.75,1.45)\end{array}$ & $\begin{array}{c}0.69 \\
(0.46,1.02)\end{array}$ & $\begin{array}{c}0.92 \\
(0.50,1.68)\end{array}$ \\
\hline Elevated OB & $\begin{array}{c}0.97 \\
(0.78,1.22)\end{array}$ & $\begin{array}{c}0.91 \\
(0.73,1.15)\end{array}$ & $\begin{array}{c}0.95 \\
(0.74,1.21)\end{array}$ & $\begin{array}{c}0.89 \\
(0.67,1.18)\end{array}$ & $\begin{array}{c}0.85 \\
(0.60,1.19)\end{array}$ & $\begin{array}{c}0.97 \\
(0.72,1.29)\end{array}$ & $\begin{array}{c}1.04 \\
(0.81,1.33)\end{array}$ & $\begin{array}{c}1.03 \\
(0.76,1.39)\end{array}$ & $\begin{array}{c}0.92 \\
(0.69,1.22)\end{array}$ & $\begin{array}{c}0.91 \\
(0.66,1.24)\end{array}$ & $\begin{array}{c}0.85 \\
(0.54,1.34)\end{array}$ \\
\hline Elevated OW & $\begin{array}{c}0.99 \\
(0.80,1.23)\end{array}$ & $\begin{array}{c}1.09 \\
(0.86,1.38)\end{array}$ & $\begin{array}{c}0.96 \\
(0.72,1.26)\end{array}$ & $\begin{array}{c}1.00 \\
(0.78,1.29)\end{array}$ & $\begin{array}{c}0.99 \\
(0.75,1.30)\end{array}$ & $\begin{array}{c}1.02 \\
(0.79,1.32)\end{array}$ & $\begin{array}{c}1.00 \\
(0.76,1.33)\end{array}$ & $\begin{array}{c}1.00 \\
(0.77,1.32)\end{array}$ & $\begin{array}{c}0.96 \\
(0.76,1.23)\end{array}$ & $\begin{array}{c}0.94 \\
(0.68,1.29)\end{array}$ & $\begin{array}{c}1.29 \\
(0.80,2.07)\end{array}$ \\
\hline Elevated OW/OB & $\begin{array}{c}0.95 \\
(0.76,1.20)\end{array}$ & $\begin{array}{c}0.99 \\
(0.76,1.29)\end{array}$ & $\begin{array}{c}0.91 \\
(0.68,1.21)\end{array}$ & $\begin{array}{c}0.89 \\
(0.66,1.19)\end{array}$ & $\begin{array}{c}0.84 \\
(0.61,1.16)\end{array}$ & $\begin{array}{c}0.99 \\
(0.77,1.27)\end{array}$ & $\begin{array}{c}1.05 \\
(0.78,1.39)\end{array}$ & $\begin{array}{c}1.03 \\
(0.75,1.41)\end{array}$ & $\begin{array}{c}0.88 \\
(0.64,1.21)\end{array}$ & $\begin{array}{c}0.84 \\
(0.60,1.17)\end{array}$ & $\begin{array}{c}1.12 \\
(0.64,1.96)\end{array}$ \\
\hline
\end{tabular}

*Reference group is No Snacks $(n=2853,13 \%)$.

**Adjusted for ethnicity, gender, age, estimated energy ratio (kcal/estimated energy requirement), poverty income ratio, Body Mass Index, Physical Activity, smoking, and Alcohol. Body Mass Index (BMI) was controlled

for all of the CVRF except for BMI.
**Statistically different from No Snacks, bolded results indicate significant findings using Bonferroni correction $p \leq 0.0042$.

${ }^{+}$Definition of elevated CVRF: Overweight/obesity was defined as a BMI $\geq 25$. Having $\geq 3$ of the following association factors: abdominal obesity, waist circumference $\geq 102 \mathrm{~cm}$ (males), $\geq 88 \mathrm{~cm}$ (females); elevated blood pressure, SBP $\geq 130 \mathrm{mmHg}$ or DBP $\geq 85 \mathrm{mmHg}$ or antihypertensive medication use; reduced serum HDL-C, $<40 \mathrm{mg} / \mathrm{dL}$ (males), $<50 \mathrm{mg} / \mathrm{dL}$ (females) or medication use for reduced HDL-C; elevated serum pressure, $\mathrm{SBP} \geq 130 \mathrm{mmHg}$ or DBP $\geq 85 \mathrm{mmHg}$ or antihypertensive medication use; reduced serum HDL-C, $<40 \mathrm{mg} / \mathrm{dL}$ (males), $<50 \mathrm{mg} / \mathrm{dL}$ (females) or medication
triacylglycerides, $\geq 150 \mathrm{mg} / \mathrm{dL}$ or medication use for elevated TAG; elevated fasting plasma or serum glucose, $\geq 100 \mathrm{mg} / \mathrm{dL}$ or medication use for elevated glucose.

Abbreviations used: BP, Blood pressure; $L D L-C$, Low-density lipoprotein cholesterol; HDL-C, High-density lipoprotein cholesterol; TAG, Triacylglycerides; WC, Waist circumference; $O B$, Obese; OW, Overweight; OW/OB, Overweight/obese; LCL, Lower Confidence Level; UCL, Upper Confidence Level. 
The Institute of Medicine's threshold of 25\% of energy [78]. The data suggest that even those adults consuming snacks with nutrient-dense foods or beverages, such as vegetables/legumes, low fat milk, and whole fruit, may need to improve aspects of their overall diet.

Snacking has been shown to contribute significantly to nutrient intake $[11,16,17]$, better diet quality [18], and to an increased likelihood of meeting selected food recommendations $[11,16]$. As shown in this study, total intake of nutrients of public health concern and nutrients underconsumed, as defined by the 2010 Dietary Guidelines for Americans [47], were higher in several of the snacking patterns when compared to no snacks.

Contrary to expectations, snacking was associated with a slightly more nutrient-dense diet. In our study, five snacking patterns had significantly higher HEI-2005 scores (when compared to no snacks). This could be a reflection that some of the foods in those snacking patterns were nutrient-dense or that poor snacking choices were compensated with healthier food/beverage choices made at the other meals. Despite the higher HEI-2005 scores found with some of the snacking patterns, the mean HEI-2005 scores for all of the snacking patterns (including no snacks) were low, suggesting that overall diet quality in adults was poor and needs improvement. These data also suggest that simply consuming a snack may not be associated with a higher diet quality, but the specific foods consumed as a snack or at meals are equally important.

Consumers may need additional educational tools on how to incorporate healthier food and beverage choices into their routine snacking behaviors. One study found that women needed information concerning snacks high in fiber and low in trans-fat [79]. Given that mothers are the primary food shoppers in the family, their lack of knowledge in selecting healthier snacks may translate into less healthier snacks being available and accessible in the homes for their children. Family-based interventions are needed for enhancing self-confidence for healthful snack selection and for overcoming barriers [79] among mothers and their children.

An important finding from this study was the lack of association between majority of the snacking patterns and weight. This is consistent with other studies [11,24-28]; however, there are studies that have shown an inverse $[19,24]$ or positive [23] association between snacking and weight. There are several possible explanations for the lack of association between snacking patterns and weight, despite the increased energy intake associated with the snacking patterns. Snacking has been shown to promote satiety and reduce risks for obesity with improved diet quality $[11,14,33]$ and increased intakes of fruit, whole grains, and fiber [11,14]. Snacking has also been associated with increased vigorous physical activity
$[16,34]$; thus, the increased energy intake associated with snacking may have been balanced with increased energy expenditure during physical activity. More studies are needed to examine whether the increased energy intake reported for those who snack is an artifact of underreporting among those who do not snack or is due to increased compensation for physical activity or a lack of compensation at subsequent meals.

To our knowledge, this is the first study to show no association between snacking and CVRF. None of the snacking patterns were associated with CVRF. Possible explanations for the lack of association between snacking patterns and CVRF are less clear. A majority of the snacking patterns exceeded the recommendation of less than $10 \%$ of total energy from SFA [47]. Dietary SFA has been shown to increase LDL-C [80], and therefore has been associated with increased risk of CVD; however, recent findings question the role of SFA in CHD risk [81]. Another possible explanation for finding no association between snacking patterns and CVRF may reflect other protective nutrients (namely calcium, potassium, fiber, folate, and magnesium) that were consumed in higher amounts in those adults who snacked compared to those who did not snack. More studies are needed to better understand the complex synergistic interactions among foods and snacking patterns in relation to health.

\section{Limitations}

NHANES is a cross-sectional study; thus, cause and effect associations cannot be inferred. Twenty-four hour dietary recalls have several inherent limitations, including that they may not reflect usual intake and are memory dependent, which may lead to under- or over-reporting; however, a single 24-hour recall is sufficient to report mean group intake [82]. Energy-dense, nutrient-poor foods and beverages, particularly when consumed as snacks, tend to be under-reported $[26,83,84]$. Data from this study suggested that the percent of overweight/ obese adults who reported $>30 \%$ of energy from snacks was significantly lower compared to normal weight adults. Whether this reflects under-reporting is debatable and needs further exploration.

\section{Conclusions}

Twelve snacking patterns (including no snacks) were identified in a nationally representative population of adults $19+$ years of age. The patterns varied in food and beverage selections and their contribution to daily intake of nutrients and diet quality. More studies are needed to confirm these findings to better understand how specific snacking patterns fit within an overall healthier eating lifestyle. Some snacking patterns may also be inversely associated with weight and abdominal obesity. Because of inconsistent evidence in the literature, there are 
several noteworthy findings from this study that should generate future hypotheses for further testing. Moreover, longitudinal studies are needed to further evaluate whether snacking prevents weight gain in adults.

\section{Additional file}

Additional file 1: Figure S1. Distribution of Percent Energy from Snacks by Weight Status for Adults $\geq 19$ Years of Age Participating in the 2001-2008 NHANES. *p $<0.001$

\section{Abbreviations}

BMI: Body mass index; BP: Blood pressure; CVRF: Cardiovascular risk factors; g: grams; HEl: Healthy eating index; HDL-C: High-density lipoprotein cholesterol; kcal: kilocalories; LDL-C: Low-density lipoprotein cholesterol; mcg: micrograms; MEC: Mobile Examination Center; mg: milligrams; MPED: MyPyramid Equivalents Database; NHANES: National Health and Nutrition Examination Survey; OB: obese; OW: Overweight; OW/ OB: Overweight/obese; SFA: Saturated Fatty Acids; TAG: Triacylglycerides; USDA: United States Department of Agriculture.

\section{Competing interests}

None of the authors have any financial or competing interests to declare.

\section{Authors' contributions}

Author contributions include the following: TN was responsible for conception and design, analysis and interpretation of data, and drafting the article. TN, CON \& VLF were responsible critical revision of paper for important intellectual content. All authors had full access to all of the data (including statistical reports and tables) in the study, can take responsibility for the integrity of the data and the accuracy of the data analysis, and approved the final version to be published.

\section{Acknowledgements}

This work is a publication of the United States Department of Agriculture (USDA/ARS) Children's Nutrition Research Center, Department of Pediatrics, Baylor College of Medicine, and Houston, Texas. The contents of this publication do not necessarily reflect the views or policies of the USDA, nor does mention of trade names, commercial products, or organizations imply endorsement from the U.S. government. This research project was supported by the USDA Agricultural Research Service through specific cooperative agreement 58-6250-0-008. Partial support was received from the USDA Hatch Project LAB 93951 and the Kellogg's Corporate Citizenship Fund. The funding agencies had no input into the study design or interpretation of the data. Special thanks to Lori Briones for help in preparing the manuscript and Bee Wong for obtaining research articles.

\section{Author details}

${ }^{1}$ Department of Pediatrics, Baylor College of Medicine, USDA/ARS Children's Nutrition Research Center, 1100 Bates Ave, Houston, TX 77030, USA. ${ }^{2}$ School of Nutrition and Food Sciences, Louisiana State University Agricultural Center, 261 Knapp Hall, 110 LSU Union Square, Baton Rouge, LA 70803, USA. ${ }^{3}$ Nutrition Impact LLC, 9725 D Drive North, Battle Creek, MI 49014, USA.

Received: 31 May 2013 Accepted: 7 April 2014

Published: 23 April 2014

\section{References}

1. Ogden CL, Carroll MD, Kit BK, Flegal KM: Prevalence of obesity in the United States, 2009-2010. NCHS Data Brief 2012, (82):1-8. http://www.ncbi. nlm.nih.gov/pubmed/22617494

2. NHLBI Obesity Education Initiative Expert Panel on the Identification, Evaluation, and Treatment of Obesity in Adults (US): Clinical Guidelines on the Identification, Evaluation, and Treatment of Overweight and Obesity in Adults: The Evidence Report. Bethesda (MD): National Heart, Lung, and Blood Institute; 1998. http://www.ncbi.nlm.nih.gov/books/NBK2003/.

3. Park YW, Zhu S, Palaniappan L, Heshka S, Carnethon MR, Heymsfield SB: The metabolic syndrome: prevalence and associated risk factor findings in the US population from the third national health and nutrition examination survey, 1988-1994. Arch Intern Med 2003, 163(4):427-436.

4. Bungum T, Satterwhite M, Jackson AW, Morrow JR Jr: The relationship of body mass index, medical costs, and job absenteeism. Am J Health Behav 2003, 27(4):456-462.

5. Fontaine KR, Redden DT, Wang C, Westfall AO, Allison DB: Years of life lost due to obesity. JAMA 2003, 289(2):187-193.

6. Ford ES, Moriarty DG, Zack MM, Mokdad AH, Chapman DP: Self-reported body mass index and health-related quality of life: findings from the behavioral risk factor surveillance system. Obes Res 2001, 9(1):21-31.

7. Sturm R, Ringel JS, Andreyeva T: Increasing obesity rates and disability trends. Health Aff (Millwood) 2004, 23(2):199-205.

8. Burton WN, Chen CY, Schultz AB, Edington DW: The costs of body mass index levels in an employed population. Stat Bull Metrop Insur Co 1999, 80(3):8-14.

9. Cawley J, Meyerhoefer C: The medical care cost of obesity: an instrumental variables approach. J Health Econ 2012, 31(1):219-230.

10. Piernas C, Popkin B: Snacking increased among U.S. adults between 1977 and 2006. J Nutr 2010, 140(2):325-332.

11. Hampl JS, Heaton CL, Taylor CA: Snacking patterns influence energy and nutrient intakes but not body mass index. J Hum Nutr Diet 2003, 16(1):3-11.

12. Piernas C, Popkin B: Trends in snacking among U.S. children. Health Aff 2010, 29(3):398-404.

13. Jahns $L$, Siega-Riz AM, Popkin BM: The increasing prevalence of snacking among US children from 1977 to 1996. J Pediatr 2001, 138(4):493-498.

14. Kerr MA, Rennie KL, McCaffrey TA, Wallace JM, Hannon-Fletcher MP, Livingstone MB: Snacking patterns among adolescents: a comparison of type, frequency and portion size between Britain in 1997 and Northern Ireland in 2005. Br J Nutr 2009, 101(1):122-131.

15. Nielsen SJ, Popkin BM: Patterns and trends in food portion sizes, 1977-1998. JAMA 2003, 289(4):450-453.

16. Kerver JM, Yang EJ, Obayashi S, Bianchi L, Song WO: Meal and snack patterns are associated with dietary intake of energy and nutrients in US adults. J Am Diet Assoc 2006, 106(1):46-53.

17. Cross AT, Babicz D, Cushman LF: Snacking patterns among 1,800 adults and children. J Am Diet Assoc 1994, 94(12):1398-1403.

18. Zizza C, Xu B: Snacking is associated with overall diet quality among adults. J Acad Nutr Diet 2012, 112(2):291-296.

19. Fabry P, Fodo J, Hejl Z, Braun T, Zvolankova K: The frequency of meals: Its relation to overweight, hypercholesterolaemia, and decreased glucose tolerance. Lancet 1964, 2(7360):614-615.

20. Larson N, Story M: A review of snacking patterns among children and adolescents: what are the implications of snacking for weight status? Child Obes 2013, 9(2):104-115.

21. Miller R, Benelam B, Stanner S, Buttriss J: Is snacking good or bad for health: an overview. Nutr Bull 2013, 38(3):302-322.

22. Booth DA: Mechanisms from models-actual effects from real life: the zero-calorie drink-break option. Appetite 1988, 11(Suppl 1):94-102.

23. Basdevant A, Craplet C, Guy-Grand B: Snacking patterns in obese French women. Appetite 1993, 21(1):17-23.

24. Drummond $\mathrm{S}$, Crombie $\mathrm{N}$, Kirk T: A critique of the effects of snacking on body weight status. Eur J Clin Nutr 1996, 50(12):779-783.

25. Keast D, Nicklas T, O'Neil C: Snacking is associated with reduced risk of overweight and reduced abdominal obesity in adolescents: NHANES 1999-2004. Am J Clin Nutr 2010, 92(2):428-435

26. Summerbell CD, Moody RC, Shanks J, Stock MJ, Geissler C: Relationship between feeding pattern and body mass index in 220 free-living people in four age groups. Eur J Clin Nutr 1996, 50(8):513-519.

27. Phillips SM, Bandini LG, Naumova EN, Cyr H, Colclough S, Dietz WH, Must A: Energy-dense snack food intake in adolescence: longitudinal relationship to weight and fatness. Obes Res 2004, 12(3):461-472.

28. Andersson I, Rossner S: Meal patterns in obese and normal weight men: the 'Gustaf' study. Eur J Clin Nutr 1996, 50(10):639-646.

29. Field AE, Austin SB, Gillman MW, Rosner B, Rockett HR, Colditz GA: Snack food intake does not predict weight change among children and adolescents. Int J Obes Relat Metab Disord 2004, 28(10):1210-1216.

30. Kant AK, Graubard BI: Secular trends in patterns of self-reported food consumption of adult Americans: NHANES 1971-1975 to NHANES 1999-2002. Am J Clin Nutr 2006, 84(5):1215-1223.

31. Howarth NC, Huang TT, Roberts SB, Lin BH, McCrory MA: Eating patterns and dietary composition in relation to $\mathrm{BMI}$ in younger and older adults. Int J Obes (Lond) 2007, 31(4):675-684. 
32. Summerbell CD, Moody RC, Shanks J, Stock MJ, Geissler C: Sources of energy from meals versus snacks in 220 people in four age groups. Eur J Clin Nutr 1995, 49(1):33-41.

33. Ovaskainen ML, Reinivuo H, Tapanainen H, Hannila ML, Korhonen T, Pakkala $H$ : Snacks as an element of energy intake and food consumption. Eur J Clin Nutr 2006, 60(4):494-501.

34. Drummond SE, Crombie NE, Cursiter MC, Kirk TR: Evidence that eating frequency is inversely related to body weight status in male, but not female, non-obese adults reporting valid dietary intakes. Int J Obes Relat Metab Disord 1998, 22(2):105-112.

35. Heidemann C, Scheidt-Nave C, Richter A, Mensink GB: Dietary patterns are associated with cardiometabolic risk factors in a representative study population of German adults. Br J Nutr 2011, 106(8):1253-1262.

36. Vergetaki A, Linardakis M, Papadaki A, Kafatos A: Presence of metabolic syndrome and cardiovascular risk factors in adolescents and University students in Crete (Greece), according to different levels of snack consumption. Appetite 2011, 57(1):278-285.

37. Yu R, Woo J, Chan R, Sham A, Ho S, Tso A, Cheung B, Lam T, Lam K: Relationship between dietary intake and the development of type 2 diabetes in a Chinese population: the Hong Kong dietary survey. Publ Health Nutr 2011, 14(7):1133-1141.

38. Oba S, Nagata C, Nakamura K, Fujii K, Kawachi T, Takatsuka N, Shimizu H: Diet based on the Japanese food guide spinning toop and subsequent mortality among men and women in a general Japanese population. J Am Diet Assoc 2009, 109(9):1540-1547.

39. Mekary RA, Giovannucci E, Willett WC, van Dam RM, Hu FB: Eating patterns and type 2 diabetes risk in men: breakfast omission, eating frequency, and snacking. Am J Clin Nutr 2012, 95(5):1182-1189.

40. National Center for Health Statistics: Analytic and Reporting Guidelines: The National Health and Nutrition Examination Survey (NHANES). 2006. Available at: http://www.cdc.gov/nchs/data/nhanes/nhanes_03_04/ nhanes_analytic_guidelines_dec_2005.pdf.

41. Moshfegh AJ, Rhodes DG, Baer DJ, Murayi T, Clemens JC, Rumpler WV, Paul DR, Sebastian RS, Kuczynski KJ, Ingwersen LA, Staples RC, Cleveland LE: The US department of agriculture automated multiple-pass method reduces bias in the collection of energy intakes. Am J Clin Nutr 2008, 88(2):324-332

42. Moshfegh A, Borrund L, Perloff B, LaComb R: Improved method for the 24-hour dietary recall for use in national surveys. FASEB J 1999, 13:A603.

43. Anand J, Goldman J, LaComb R: An approved approach to 24-hour dietary recall methodology. Ann Nutr Metab 2001, 45(Suppl 1):156.

44. Conway JM, Ingwersen LA, Vinyard BT, Moshfegh AJ: Effectiveness of the US department of agriculture 5-step multiple-pass method in assessing food intake in obese and nonobese women. Am J Clin Nutr 2003, 77(5):1171-1178

45. Centers for Disease Control and Prevention: NHANES Dietary Intenview Component. www.cdc.gov/nchs/data/nhanes/nhanes_09_10/dietaryrecall_f.pdf

46. U.S. Department of Agriculture, Agricultural Research Service: USDA Food and Nutrient Database for Dietary Studies, Versions 1.0 - 4.1 Documentation and User Guide, 2004, 2006, and 2008. http://www.ars.usda.gov/Services/ docs.htm?docid $=12089$.

47. United States Department of Agriculture: Center for Nutrition Policy and Promotion. Report of the Dietary Guidelines Advisory Committee on the Dietary Guidelines for Americans. 2010. Available at: http://www.cnpp. usda.gov/DGAs2010-DGACReport.htm.

48. Friday J, Bowman S: MyPyramid Equivalents Database for USDA Survey Food Codes, 1994-2002 Version 1.0. 2006. http://www.barc.usda.gov/ bhnrc/cnrg.

49. Bowman SA, Friday JE, Moshfegh A: MyPyramid Equivalents Database, 2.0 for USDA Survey Foods, 2003-2004 Food Surveys Research Group. 2006. http://www.ars.usda.gov/ba/bhnrc/fsrg.

50. U.S. Department of Agriculture, Center for Nutrition Policy and Promotion: Healthy Eating Index-2005 Development and Evaluation Technical Report Support Files. Available at: http://www.cnpp.usda.gov/Healthy Eatinglndex-2005report.htm.

51. Kennedy E, Ohls J, Carlson S, Fleming K: The healthy eating index: design and applications. J Am Diet Assoc 1995, 95(10):1103-1108.

52. Guenther PM, Reedy J, Krebs-Smith SM: Development of the healthy eating index-2005. J Am Diet Assoc 2008, 108(11):1896-1901.

53. Guenther PM, Reedy J, Krebs-Smith SM, Reeve BB: Evaluation of the healthy eating index-2005. J Am Diet Assoc 2008, 108(11):1854-1864.
54. United States Department of Agriculture: Center for Nutrition Policy and Promotion. Calculation of the Healthy Eating Index-2005 component and total scores for a population, subpopulation, or group. Available at: www.cnpp.usda.gov/Publications/HEl/HEl-2005/Readme_HEl2005_ NHANES0102PopulationScore.doc. (Accessed 12/20/13).

55. National Health and Nutrition Examination Survey: Physical Activity and Physical Fitness. http://www.cdc.gov/nchs/data/nhanes/nhanes_07_08/ paq07_08_eng.pdf.

56. Centers for Disease Control and Prevention: National Center for Health Statistics. National Health and Nutrition Examination Survey. Anthropometry Manual. http://www.cdc.gov/nchs/data/nhanes/bm.pdf.

57. National Cholesterol Education Program: National Heart, Lung, and Blood Institute. National Institutes of Health. Detection, Evaluation, and Treatment of High Blood Cholesterol in Adults (Adult Treatment Panel III). http://www.nhlbi.nih.gov/about/ncep/.

58. Centers for Disease Control and Prevention: National Center for Health Statistics. National Health and Nutrition Examination Survey (NHANES). Physician Examination Procedures Manual. Available at: http://www.cdc. gov/nchs/data/nhanes/nhanes_07_08/manual_pe.pdf.

59. Centers for Disease Control and Prevention: National Center for Health Statistics, NHANES. Laboratory Methods. http://www.cdc.gov/nchs/nhanes/ nhanes1999-2000/lab99_00.htm, http://www.cdc.gov/nchs/nhanes/nhanes20012002/lab01_02.htm, http://www.cdc.gov/nchs/nhanes/nhanes2003-2004/ lab03_04.htm, http://www.cdc.gov/nchs/nhanes/nhanes2005-2006/ lab_methods_05_06.htm http://www.cdc.gov/nchs/data/nhanes/gendoc.pdf.

60. National Institutes of Health, National Heart Lung and Blood Institute: Clinical Guidelines on the Identification, Evaluation, and Treatment of Overweight and Obesity in Adults. Obes Res 1998, 6(Suppl 2):51S-209S.

61. Grundy SM, Cleeman JI, Daniels SR, Donato KA, Eckel RH, Franklin BA, Gordon DJ, Krauss RM, Savage PJ, Smith SC Jr, Spertus JA, Costa F: Diagnosis and management of the metabolic syndrome: an American heart association/national heart, lung, and blood institute scientific statement. Circulation 2005, 112(17):2735-2752.

62. Quon MJ: Limitations of the fasting glucose to insulin ratio as an index of insulin sensitivity. J Clin Endocrinol Metab 2001, 86(10):4615-4617.

63. International Expert Committee: International Expert Committee report on the role of the A1C assay in the diagnosis of diabetes. Diabetes Care 2009, 32(7):1327-1334.

64. Dinavahi R, Falkner B: Relationship of homocysteine with cardiovascular disease and blood pressure. J Clin Hypertens (Greenwich) 2004, 6(9):494-498. quiz 499-500.

65. National Institutes of Health. National Heart, Lung, and Blood Institute: National Cholesterol Education Program (2001) Adult Treatment Panel III Executive Summary Report. http://www.nhlbi.nih.gov/guidelines/ cholesterol/atp3_rpt.htm.

66. Development and Evaluation of the Healthy Eating Index-2005: Technical Report. Center for Nutrition Policy and Promotion, U.S. Department of Agriculture. 2007. http://www.cnpp.usda.gov/HealthyEatinglndex.htm.

67. Bhupathiraju SN, Tucker KL: Coronary heart disease prevention: nutrients, foods, and dietary patterns. Clin Chim Acta 2011, 412(17-18):1493-1514.

68. Kant AK: Dietary patterns: biomarkers and chronic disease risk. Appl Physiol Nutr Metab 2010, 35(2):199-206.

69. Tucker KL: Dietary patterns, approaches, and multicultural perspective. Appl Physiol Nutr Metabol Physiol Appl Nutr Metabol 2010, 35(2):211-218.

70. Judd SE, Gutierrez OM, Newby PK, Howard G, Howard VJ, Locher JL, Kissela BM, Shikany JM: Dietary patterns are associated with incident stroke and contribute to excess risk of stroke in black Americans. Stroke 2013, 44(12):3305-3311. http://www.ncbi.nlm.nih.gov/pubmed/24159061.

71. Kimokoti RW, Gona P, Zhu L, Newby PK, Millen BE, Brown LS, D'Agostino RB, Fung TT: Dietary patterns of women are associated with incident abdominal obesity but not metabolic syndrome. J Nutr 2012, 142(9):1720-1727. http://www.ncbi.nlm.nih.gov/pubmed/22833658.

72. Liu E, McKeown NM, Newby PK, Meigs JB, Vasan RS, Quatromoni PA, D'Agostino RB, Jacques PF: Cross-sectional association of dietary patterns with insulin-resistant phenotypes among adults without diabetes in the Framingham Offspring Study. Br J Nutr 2009, 102(4):576-583.

73. Newby PK, Tucker KL: Empirically derived eating patterns using factor or cluster analysis: a review. Nutr Rev 2004, 62(5):177-203.

74. Wang L, Lee IM, Manson JE, Buring JE, Sesso HD: Alcohol consumption, weight gain, and risk of becoming overweight in middle-aged and older women. Arch Intern Med 2010, 170(5):453-461. 
75. Lopez-Garcia E, Van Dam RM, Rajpathak S, Willett WC, Manson JE, Hu FB: Changes in caffeine intake and long-term weight change in men and women. Am J Clin Nutr 2006, 83(3):674-680.

76. Mohindra NA, Nicklas TA, O'Neil CE, Yang SJ, Berenson GS: Eating patterns and overweight status in young adults: the Bogalusa Heart Study. Int J Food Sci Nutr 2009, 60(Suppl 3):14-25.

77. Nicklas TA, Yang SJ, Baranowski T, Zakeri I, Berenson G: Eating patterns and obesity in children: the Bogalusa Heart Study. Am J Prev Med 2003, 25(1):9-16.

78. Food and Nutrition Board: Dietary Reference Intakes for Energy, Carbohydrate, Fiber, Fat, Fatty Acids, Cholesterol, Protein, and Amino Acids. http://iom.edu/Reports/2002/Dietary-Reference-Intakes-for-EnergyCarbohydrate-Fiber-Fat-Fatty-Acids-Cholesterol-Protein-and-Amino-Acids. aspx.

79. Schunk J, McArthur L, Maahs-Fladung C: Correlates for healthful snacking among middle-income Midwestern Women. J Nutr Educ Behav 2009, 41:274-280.

80. Siri-Tarino P, Sun Q, Hu F, Krauss R: Saturated fatty acids and risk of coronary heart disease: modulation by replacement nutrients. Curr Atherosclerosis Rep 2010, 12(6):384-390.

81. Astrup A, Dyerberg J, Elwood P, Hermansen K, Hu FB, Jakobsen MU, Kok FJ, Krauss RM, Lecerf JM, LeGrand P, Nestel P, Riserus U, Sanders T, Sinclair A, Stender S, Tholstrup T, Willett WC: The role of reducing intakes of saturated fat in the prevention of cardiovascular disease: where does the evidence stand in 2010? Am J Clin Nutr 2011, 93(4):684-688.

82. Thompson FE, Byers T: Dietary assessment resource manual. J Nutr 1994, 124(11 Suppl):2245S-2317S.

83. Poslusna K, Ruprich J, de Vries J, Jakubikova M, van't Veer P: Misreporting of energy and micronutrient intake estimated by food records and 24 hour recalls, control and adjustment methods in practice. Br J Nutr 2009, 101(Suppl 2):S73-S85.

84. Champagne CM, Baker NB, DeLany JP, Harsha DW, Bray GA: Assessment of energy intake underreporting by doubly labeled water and observations on reported nutrient intakes in children. J Am Diet Assoc 1998, 98(4):426-433.

\section{Submit your next manuscript to BioMed Central and take full advantage of:}

- Convenient online submission

- Thorough peer review

- No space constraints or color figure charges

- Immediate publication on acceptance

- Inclusion in PubMed, CAS, Scopus and Google Scholar

- Research which is freely available for redistribution 